\title{
Controlled Growth of Ultrathin Film of Organic Semiconductors by Balancing the Competitive Processes in Dip-Coating for Organic Transistors
}

Kunjie $W u,{ }^{\dagger}, \perp$ Hongwei Li, ${ }^{\dagger}, \perp$ Liqiang $L i^{\dagger,}$ Suna Zhang, ${ }^{\dagger}$ Xiaosong Chen ${ }^{\dagger}$ Zeyang Xu, ${ }^{\dagger}$ Xi Zhang, ${ }^{\dagger}$ Wenping $\mathrm{Hu},{ }^{\dagger,},{ }^{*}$ Lifeng Chi, ${ }^{\S,}{ }^{*}$ Xike Gao, ${ }^{\text {l, * }}$ and Yancheng Meng,

*lqli2014@sinano.ac.cn, *huwp@tju.edu.cn, ${ }^{*}$ chilf@suda.edu.cn, ${ }_{\text {*gaoxk@sioc.ac.cn }}^{*}$

(1) Growth results of TIPS-pentacene in single solvent system

Table S1. Viscosity and surface tension of several common solvents ${ }^{\mathrm{a}}$

\begin{tabular}{|l|l|l|}
\hline Solvent & Viscosity $(\mathrm{mPa} \cdot \mathrm{s})$ & Surface tension $(\mathrm{mN} / \mathrm{m})$ \\
\hline toluene & -0.257 & 27.9 \\
\hline chloroform & -0.271 & 26.5 \\
\hline octane & -0.288 & 21.2 \\
\hline acetone & -0.519 & 22.7 \\
\hline ethanol & 0.035 & 21.9 \\
\hline
\end{tabular}

${ }^{\text {a }}$ G. W. Kauffman, P. C. Jurs, J. Chem. Inf. Comput. Sci. 2001, 41, 408-418.

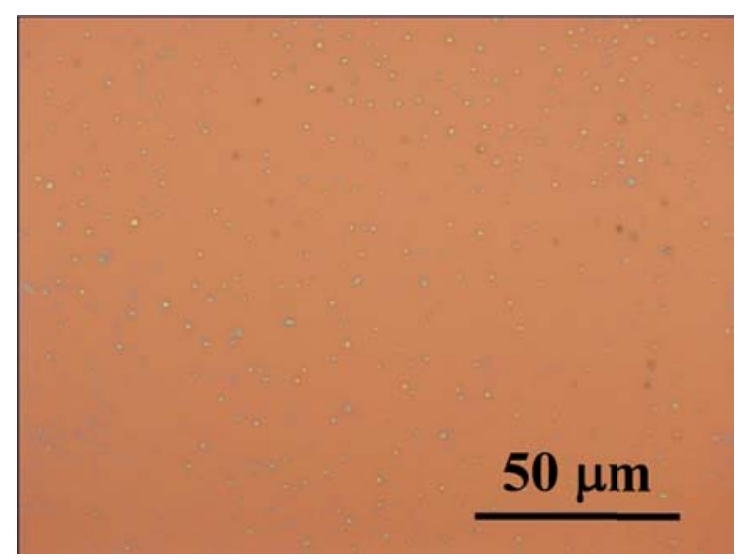

Figure S1. Sparse dots of TIPS-pentacene grown in toluene solution $(4 \mathrm{mg} / \mathrm{ml})$ at a pulling speed of $500 \mathrm{um} / \mathrm{s}$. 

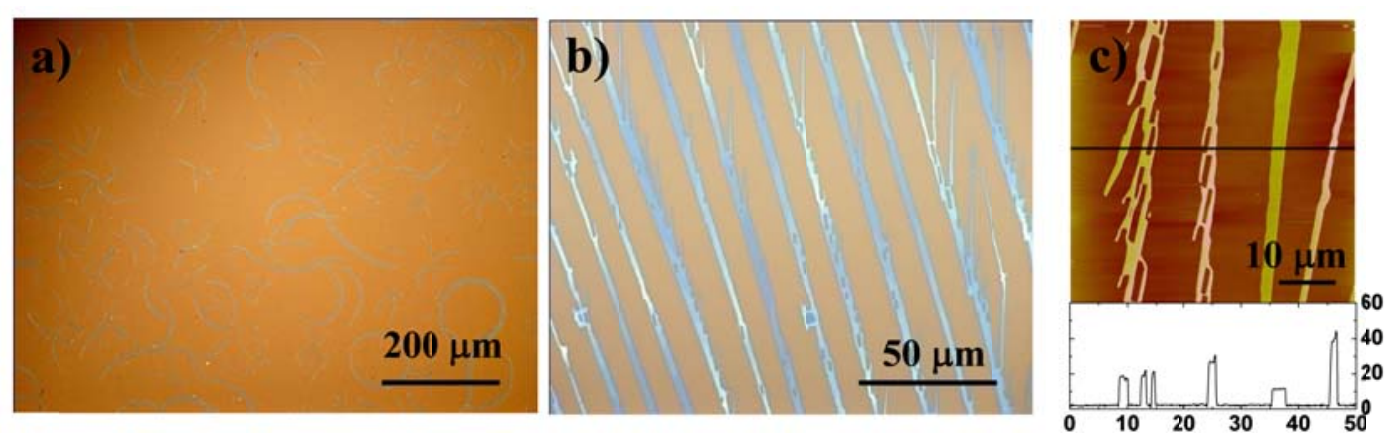

Figure S2. (a) Discontinuous microstripes of TIPS-pentacene grown in octane solution $(2 \mathrm{mg} / \mathrm{ml})$ at a pulling speed of $200 \mathrm{um} / \mathrm{s}$. (b) Optical images and (c) AFM image and section profile of non-uniform microstripes of TIPS-pentacene grown in octane solution $(2 \mathrm{mg} / \mathrm{ml})$ at a pulling speed of $2000 \mu \mathrm{m} / \mathrm{s}$. The unit of $\mathrm{x}, \mathrm{y}$-axis in section profile (c) is $\mu \mathrm{m}$ and $\mathrm{nm}$, respectively.
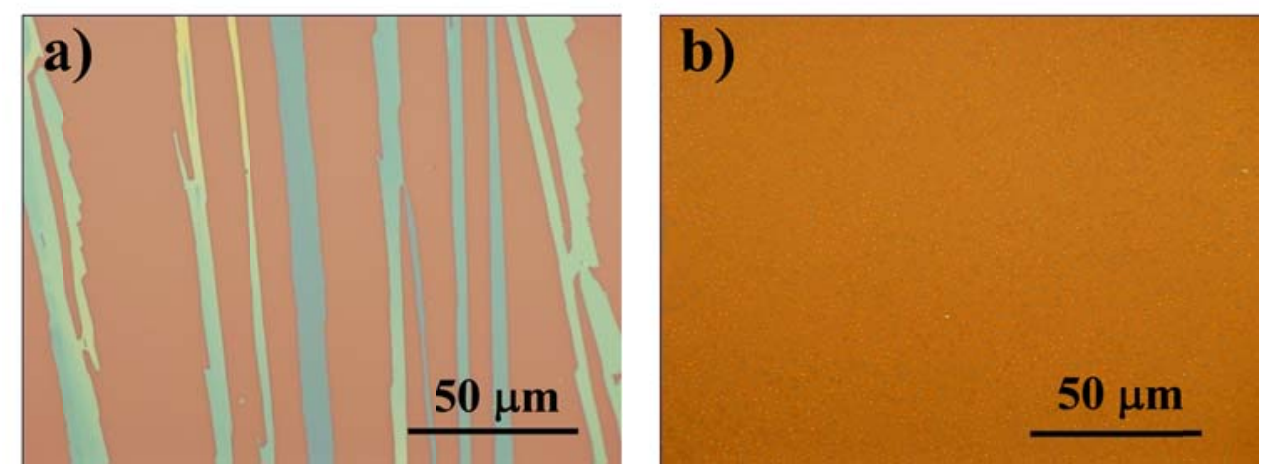

Figure S3. (a) Non-uniform microstripes of TIPS-pentacene in hexane solution (1.6 $\mathrm{mg} / \mathrm{ml}$ ) at $10 \mathrm{um} / \mathrm{s}$. (b) Dots of TIPS-pentacene in hexane solution $(1.6 \mathrm{mg} / \mathrm{ml})$ at $1000 \mathrm{um} / \mathrm{s}$.
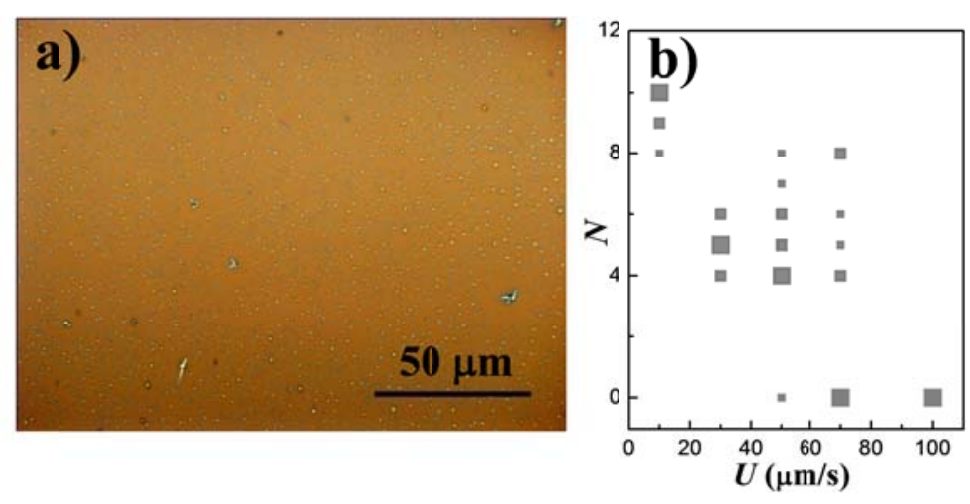

Figure S4. Growth results of of TIPS-pentacene in acetone solution, (a) optical image of discontinuous dots obtained at $100 \mu \mathrm{m} / \mathrm{s}$. (b) Relationship between molecular layer number $(N)$ and pulling speed $(U)$. The number of " 0 " denotes discontinuous dots. 


\section{(2) Growth results of TIPS-pentacene in mixed solvent system}

The mixed solvent with toluene as good solvent always yield no ultrathin film at low concentration, which may be due to toluene's big solubility for TIPS-pentacene and relatively low volatility.

For chloroform, hexane is first used as poor solvent to lower the solubility for TIPS-pentacene, and a mixture of chloroform:hexane (2:9) is used to prepare the TIPS-pentacene solution with concentration of $1.6 \mathrm{mg} / \mathrm{mL}$. Low speed regime yields microstripes with thickness over $15 \mathrm{~nm}$ (Figure S5), while discontinuous dots form at high speed regime. It has been already demonstrated that uniform ultrathin film can be obtained in the entrainment controlled regime at high speed range (Figure 2a, right part, in maintext). However, no uniform ultrathin film is obtained in this system at high speed regime, which may be related to the fast evaporation rate of both chloroform and hexane.

Based on this analysis, isopropanol with relatively low volatility is used as poor solvent to form mixed solvent (chloroform:isopropanol, 1:2). As expected, at high speed range (200-1000 $\mu \mathrm{m} / \mathrm{s}$ ), entrainment can be found on the substrate (Figure S6), and uniform dentritic ultrathin film with thickness less than $10 \mathrm{~nm}$ (Figure 4g,h, in maintext) forms over large area (over hundreds of $\mu \mathrm{m}$ ) at this regime. On the other hand, at low pulling speed of $10 \mu \mathrm{m} / \mathrm{s}$, very thick microstripes form in the meniscus controlled regime. 

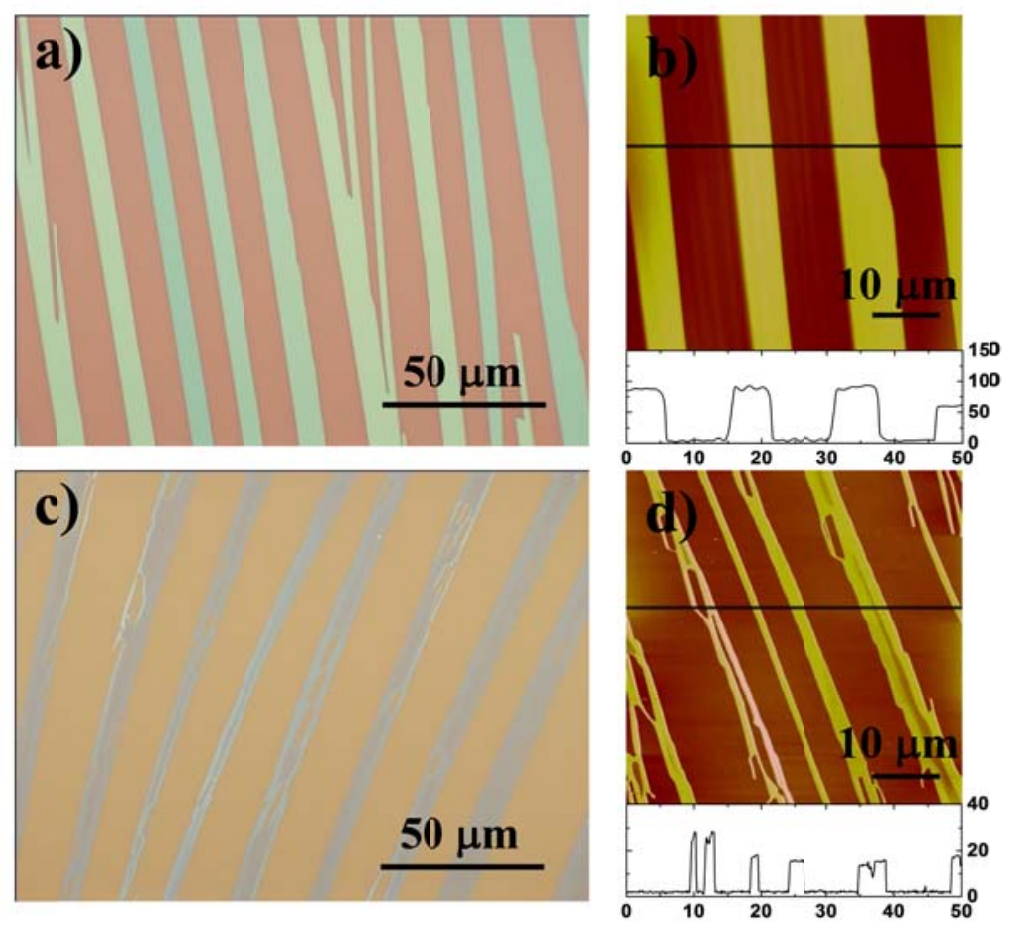

Figure S5. Growth of results of TIPS-pentacene in hexane:chloroform solution (1.6 $\mathrm{mg} / \mathrm{ml}$ ) (a) Optical image and (b) AFM image and section profile of thick microstripes at $10 \mathrm{um} / \mathrm{s}$. (c) Optical image and (d) AFM image and section profile of thick microstripes at $80 \mathrm{um} / \mathrm{s}$. The unit of $x, y$-axis in section profile $(b, d)$ is $\mu \mathrm{m}$ and nm, respectively.

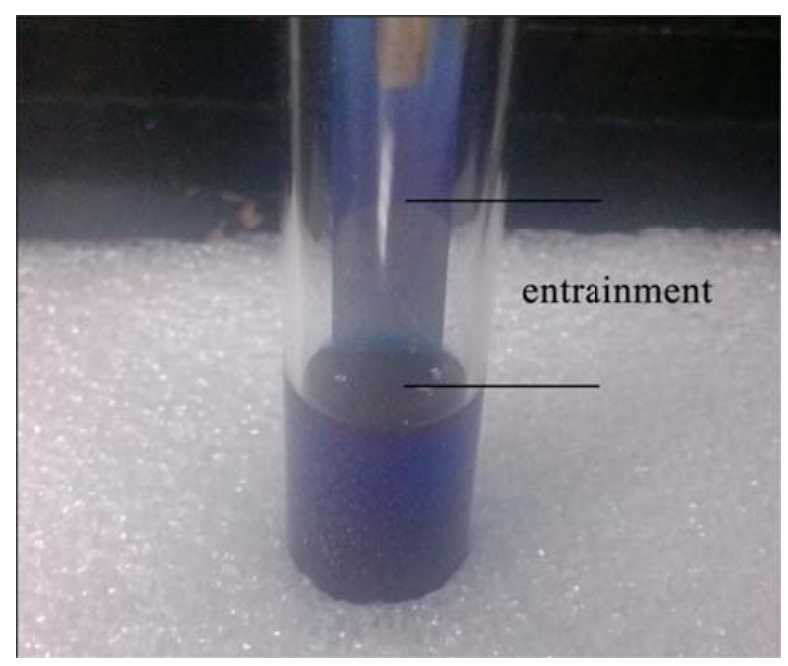

Figure S6. Optical image of dip-coating process in isopropanol:chloroform solution, showing entrainment film.

(3) XRD results of ultrathin film 

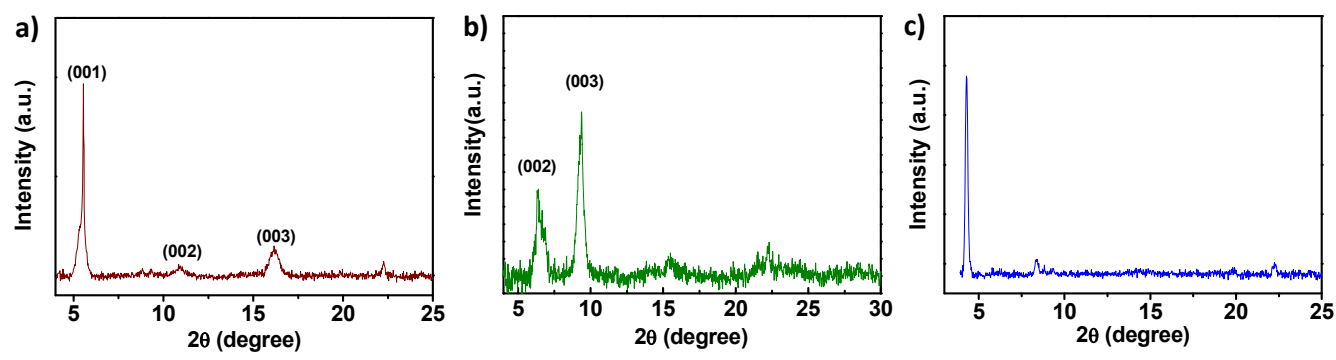

Figure S7. XRD patterns of ultrathin film of (a) TIPS-pentacene, (b) C8-BTBT, and (c) NDI3HU-DTYM2.

To testify the molecular ordering of these ultrathin film, XRD measurements with $\theta-2 \theta$ scan mode were performed (Figure S7). TIPS-pentacene and C8-BTBT microstripes and NDI monolayer with some islands yield clear XRD patterns, indicating the molecular ordering within ultrathin film. Since the crystal structure of NDI3HU-DTYM2 has not been reported so far, it is difficult to index the XRD patterns. Ultrathin microstripes of C60 does not yield clear XRD patterns.

\section{(4) Calculation of concentration range for the growth of ultrathin film}

The entrainment-controlled regime is taken as example. Generally, the thickness $(h)$ of liquid entrainment film on the substrates can be approximately predicted by Landau-Levich equation: [L. Landau, B. Levich, Acta Physicochim. URSS 1942, 17, 42]

$h=0.944(\mu U)^{2 / 3} / \sigma^{1 / 6}(\rho g)^{1 / 2}$

where $\rho, \mu$, and $\sigma$ are the density, viscosity, and surface tension of the solution, respectively, and $g$ is the gravity acceleration.

Octane is taken as example. The $\rho\left(788 \mathrm{~kg} / \mathrm{m}^{3}\right), \mu\left(2.88 \times 10^{-4} \mathrm{~N} . \mathrm{s} / \mathrm{m}\right)$, and $\sigma$ $\left(2.12 \times 10^{-2} \mathrm{~N} / \mathrm{m}\right)$ of octane are used for the calculation. The pulling speed is set in the range of $1000 \mu \mathrm{m} / \mathrm{s}$. Then, based on the Landau-Levich equation, the theoretical 
thickness of an entrained liquid film ( $\left.T_{\text {theo,liquid }}\right)$ can be approximately calculated to about $0.892 \mu \mathrm{m}$, respectively.

Then, the theoretical thickness of solute film $\left(T_{\text {theo,film }}\right)$ after solvent evaporation can be further calculated approximately by $T_{\text {theo,film }}=\left(T_{\text {theo,liquid }} \times C\right) / \rho_{\text {film }}$, where $C$ is solution concentration $(1 \mathrm{mg} / \mathrm{ml})$, and $\rho_{\text {film }}$ is the density of solute film. $T_{\text {theo,film }}$ is the thickness of full-covered solute film. In our case, the structured film is generally obtained, so we use $50 \%$ as an averaged coverage. If we assume $T_{\text {theo,film }}$ to be $1-5 \mathrm{~nm}$, and the thickness of structured film with about $50 \%$ coverage is about $2-10 \mathrm{~nm}$. The density of solute (organic semiconductors with molecular weight of about hundreds of $\mathrm{g} / \mathrm{mol}$ ) is generally about $1 \mathrm{~g} / \mathrm{cm}^{3}$. Therefore, the concentration range will be derived to be about 1.1-5.6 $\mathrm{mg} / \mathrm{mL}$, which indicates that low concentration is favorable for the growth of ultrathin film. However, the theoretical calculation will certainly have some deviation from the real situation. In view of our experiments, we conclude that 0.5-4 $\mathrm{mg} / \mathrm{mL}$ is generally favorable for the growth of ultrathin film. However, this reference range is not fixed for all the system, which may have a little variation depending on the experimental situations.

\section{(5) Electrical curves of C60 FETs}

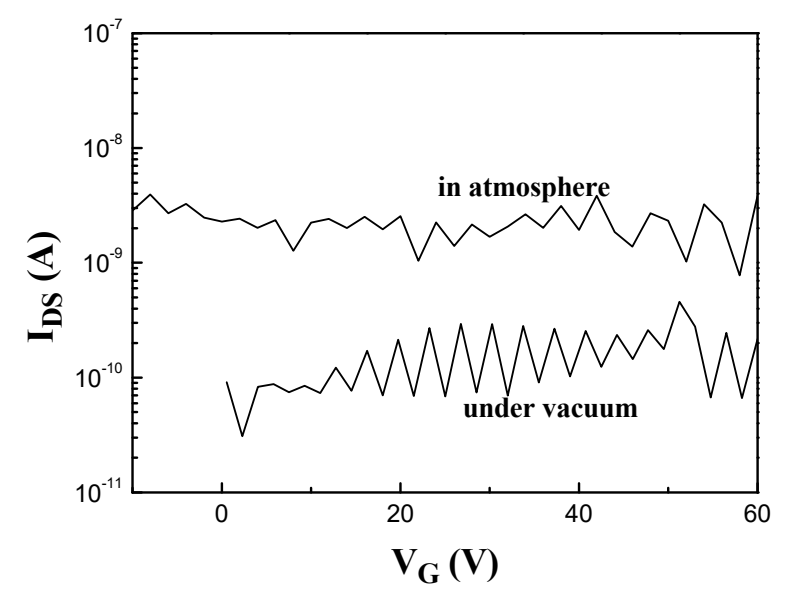

Figure S8. Transfer curves of C60 FET measured in air and under vacuum, showing no field-effect property. 
C60 FETs have been measured in air and under vacuum of $10^{-4} \mathrm{~Pa}$, but no clear n-type FET signal can be observed. Two possible reasons might account for this result: (1) the $\mathrm{C} 60 \mathrm{FET}$ is built on the bare $\mathrm{SiO}_{2}$ surface, it is known that $\mathrm{Si}-\mathrm{OH}$ group serves as the electron trap for n-type transporting [Nature 2005, 434, 194-199]; (2) the low ordering of C60 ultrathin film might be another reason for the absence of n-type signal. 